\title{
Vergence Control of Binocular Stereoscopic Camera Using Disparity Information
}

\author{
Ki-Chul Kwon, Young-Tae Lim, and Nam Kim* \\ College of Electrical and Computer Engineering, Chungbuk National University, \\ Cheong-Ju 361-763, Korea \\ Young-Jun Song \\ BITRC, Chungbuk National University, Cheong-Ju 361-763, Korea \\ Young-Soo Choi \\ Korea Atomic Energy Research Institute, Daejeon 305-353, Korea
}

(Received April 22, 2009 : revised June 22, 2009 : accepted June 22, 2009)

\begin{abstract}
The vergence control of binocular stereoscopic camera is the most essential factor for acquiring high quality stereoscopic images. In this paper, we proposed a binocular stereoscopic camera vergence control method using disparity information by the simple image processing and estimate the quantity of vergence control using the Lagrange interpolation equation. The method of extracting disparity information through image processing is as follows: first the key-object in left \& right images was extracted through labeling of the central area of the image, and then a simple method was used for calculating the disparity value of the same key-object in the labeled left and right images. The vergence control method uses disparity information and keeps the convergence distance of left \& right cameras and the distance of the key-object the same. According to the proposed method, variance in the distance of the key-object and application of calculated disparity information of obtained left \& right images to the quadratic Lagrange interpolation equation could estimate the quantity of vergence control, which confirmed that the method of stereoscopic camera vergence control can be simplified through experiments on various key-objects and other convergence distance.
\end{abstract}

Keywords : Vergence control, Disparity, Stereoscopic camera, Three-dimensional image processing

OCIS codes: (100.6890) Three-dimensional image processing; (330.4150) Motion detection; (330.7320) Vision adaptation

\section{INTRODUCTION}

Human beings repeat focus control of the thickness of the eye lens, according to the distance, and vergence control, which always keeps the key-object formed in the center of both the retinas and so, provides evernatural and clear image information[1-5]. The multiple cameras with two or more sensors or a lens array composed of many lenses are used to get three-dimensional data [6-8]. The last goal of research on stereoscopic images is to make an artificial visual device which acquires information as similar as possible to the visual

*Corresponding author: namkim@chungbuk.ac.kr information formed on both the human eyes. Acquiring a stereoscopic image similar to image information provided through both human eyes requires preceding research on the binocular stereoscopic camera method using two cameras.

There are two methods for acquiring stereoscopic images: one is the binocular stereoscopic camera method using left and right cameras grouped according to the number of cameras used, and the other is the multi view camera method for acquiring images information of a specific image concurrently from several angles. The latter can maximize the effect of stereoscopic images of limited region or object, but has some trouble in processing complex images or in moving cameras 
toward moving objects or in accurately photographing in a complex space.

For stereoscopic image by stereoscopic camera, acquiring a stereoscopic image similar to a real image first requires vergence control of left and right cameras over the key-object. The vergence control of stereoscopic cameras can be divided into the toed-in type and the parallel type[8].

There are two kinds of vergence control methods of binocular stereoscopic cameras: one is by calculating the distance information of the key-object and the other by using disparity information between left and right images according to the position of the key-object in the image. The former requires complex calculation of the mechanism by mechanical methods, and has a defect that the distance of the key-object lessening the accuracy of calculation. On the other hand, the latter has simple and accurate vergence control by using disparity information between key-objects in acquired left and right images.

As preceding research on the binocular disparity extraction method for the vergence control of binocular stereoscopic camera, Krotkov extracts the mean value and the variance value and takes advantage of the depth distribution of specific points, but the method has some trouble in accurate vergence control except in specific environments[9]. M.Tanaka attempted the vergence control of binocular stereoscopic camera using ZDF(Zero Disparity Filter) which takes advantage of left and right image edge detection[10]. H. Hirschmuller proposed a cross correlation error function for vergence control of one point. Its method of extracting disparity value by $\mathrm{ZDF}$ and correlation is also low in accuracy and has a long processing time[11]. D. J. Coombs, etc. proposed the real-time vergence control system using a Cepstral filter for estimating the quantity of vergence control error in the acquired left \& right stereoscopic images.[12-14] Last, K. C. Kwon[1] showed the theory of relationship between vergence and focus according to the object distance of parallel stereoscopic cameras, and took advantage of one-dimensional Cepstral Filter with the vertical projection data of a pair of stereoscopic images as input data for fast, accurate simultaneous control of vergence and focus. The methods of extracting disparity information that have been proposed for the vergence control of stereoscopic cameras have long-time processing and low accuracy, and also most of them are vergence control methods of toed-in stereoscopic cameras.

This study has extracted disparity information by image processing, and then proposed a vergence control method of a binocular stereoscopic camera using the former. The vergence control method proposed by this study extracts the key-object from stereoscopic data through labeling, calculates disparity information, always keeps the distance of the key-object and the convergence distance of left \& right camera the same by using the disparity information. The quantity of vergence control can be estimated by applying the disparity information calculated from the acquired left and right images to the 2nd Lagrange approximated equation while the distance of the key object is made to vary, and accordingly, the experiments confirmed that it is possible to simplify the vergence control method of the stereoscopic camera.

The proposed vergence control method can make extraction of disparity information simple and fast, and also, can make vergence control accurate, and the distance information of the key-object calculated by the focus information of the lens used can estimate the quantity of vergence control by the Lagrange approximated equation, and so, can automate the vergence control of binocular stereoscopic camera quite simply.

\section{CHARACTERISTICS OF VERGENCE CONTROL OF BINOCULAR STEREOSCOPIC CAMERA}

According to the distance of the key-object, there are two methods of binocular stereoscopic camera with controllable vergence: toed-in mode and parallel mode. The former is a binocular stereoscopic camera method controlling vergence by rotating the optic axis of the camera at an angle of $\theta$ according to the distance of the key-object as in Fig. 1(a). Parallel mode, however, is a binocular stereoscopic camera method of controlling vergence by horizontally moving separated lens and CCD sensor into symmetric position to each other. Either of them can easily acquire the quantity of vergence control $\theta$ and $h$ by interpreting their own geometric structure[1-3]. With the distance between lens and key-object as $p$, the distance between CCD sensor and lens as f, and the distance between left and right camera as $2 t$, the vergence control angle of toed-in camera $(\theta)$ and the control quantity of parallel camera (h) can be expressed as follows:
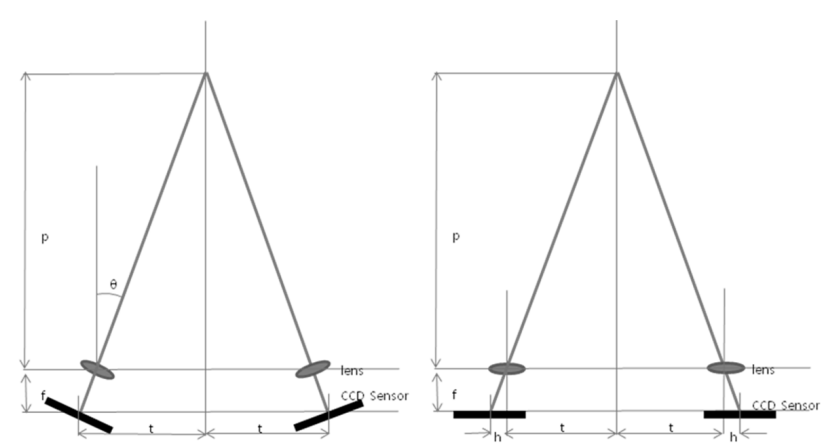

FIG. 1. Geometry of binocular stereoscopic camera of (a) Toed-in binocular stereoscopic camera and (b) Parallel binocular stereoscopic camera. 


$$
\theta=\tan ^{-1} \frac{t}{f+p}, h=\frac{f t}{p}
$$

If it is considered as there is no vertical disparity of the left and right camera for making stereoscopic camera, there always exists horizontal disparity of the stereoscopic images acquiring as the distance of the key-object. Therefore, it is just needed the process to extract disparity information of the key-object acquiring from the left and right stereoscopic images for vergence control.

\section{VERGENCE CONTROL OF BINOCULAR STEREOSCOPIC CAMERA}

\section{Automatic vergence control method by disparity information}

The proposed vergence control method of a binocular stereoscopic camera by disparity information, when the early convergence distance of the stereoscopic camera doesn't correspond to the distance of the key-object as in Fig. 2, extracts the horizontal disparity of key-object from the acquired left \& right image, and minimizes the difference between the distance of a key-object and the convergence distance of camera by keeping disparity value to the minimum [1].

The proposed vergence control algorithm of a binocular stereoscopic camera has consisted of extracting the key-object from the acquired left \& right images, as in Fig. 2, by simple image processing, and then calculating disparity information and then compensating it.

Extraction of disparity information by image processing is composed of three steps: converting color image to gray image, labeling, and extracting the key-object.

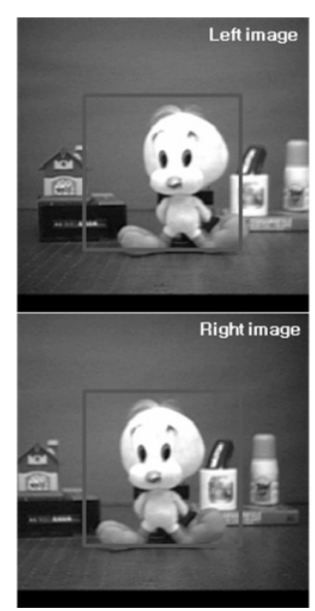

(a)

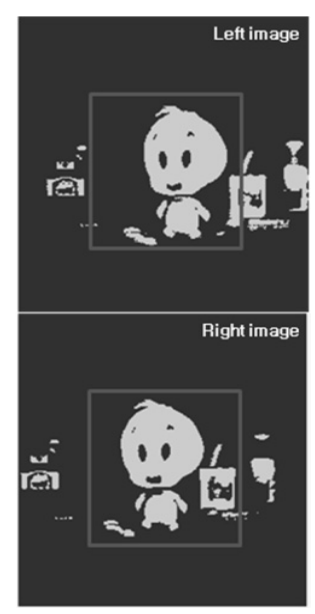

(b)

Conversion to gray image is the previous process for speeding the processing, and labeling the previous process for extracting the key-object. Fig. 3 shows an example of extracting the disparity information of the key-object through image processing. The position of the key-object and the square region in Fig. 3(a), (b) were assumed to remain in the center of the image, and the key-object was extracted within half the full region from the center of the image, and disparity information was calculated. Also, for extracting the key-object, the threshold value is fixed by applying the empirical rule to the histogram distribution of the brightness of the region of interest as in equation (2) within the labeled region through the labeling method

[15]. That is, $95 \%$ of the total brightness distribution of the region of interest is fixed as threshold value and then labeled. Threshold value $A_{t h}$ is what is the result of subtracting twice the standard deviation(s) from the

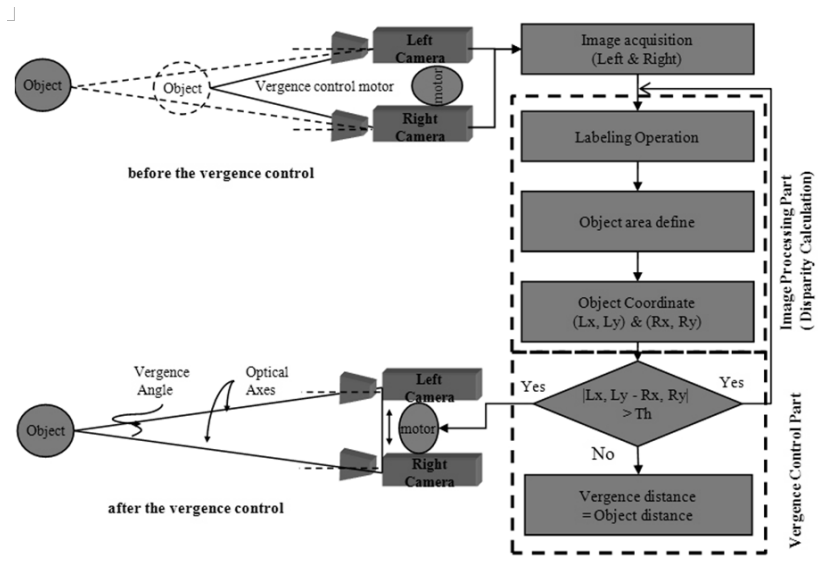

FIG. 2. Vergence control of binocular stereoscopic camera by disparity information.

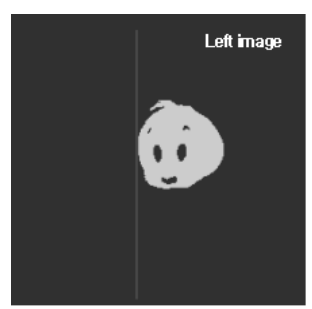

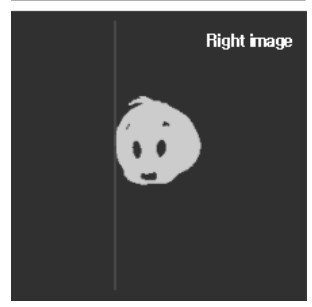

(c)

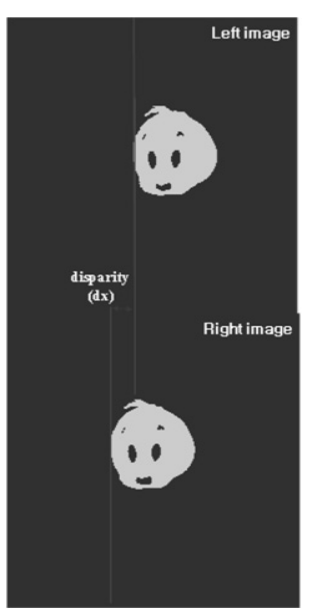

(d)

FIG. 3. Extracting binocular stereoscopic disparity information : (a) Original image. (b) Labeling. (c) Extracting left and right object. (d) Extracting the disparity information of the key-object. 
brightness value distribution of the region of interest or of adding twice the standard deviation(s) to mean value $(\bar{x})$, and the threshold value are two. Of these, whether to use the threshold value with large or small brightness is decided by considering the brightness of the key-object selectively.

$$
A_{t h}=\bar{x} \pm 2 s
$$

Fig. 3(c) shows the result of extracting the key-object by labeling left and right images. Extracting the disparity information of the key-object fixes the symbol by acquiring the coordinates of the boundary point of the key-object in left and right images, and calculates the absolute value of the difference between coordinate values. Fig. 3(d) shows an example of the result of extracting the disparity information of the test image.

\section{Automatic vergence control by estimating disparity}

For a fixed binocular stereoscopic camera, binocular disparity varies from plus to minus or from minus to plus, according to variance in the distance of the key-object. So analyzing the absolute value of binocular (left and right) distance difference according to distance between camera and key-object can estimate disparity information, and execute vergence control. That is, this can be expressed in equation (2), and its answer value is the point when the vergence control of the key-object comes to an end. The method proposed here of solving equation (2) is relatively quick and simple, and uses the Lagrange interpolation method which can be used with no relation to the interval of data.

Generally the definition of interpolation is as follows: Suppose that there is a non-polynomial function $[f(x)]$ with real value in $\mathrm{n}+1$ points $\left(x_{0}, x_{1}, \cdots, x_{n}\right)$ and a closed interval including these points on the $X$ axis, and that there is the n-th polynomial equation (4), which is called the interpolation polynomial, satisfying equation (3) of these points. And interpolation means finding the approximated value of $f(x)$ other than the given points by interpolation polynomial.

$$
\begin{aligned}
& p\left(x_{i}\right)=f\left(x_{i}\right), i=0,1, \ldots, n \\
& p(x)=a_{n} x_{n}+a_{n-1} x_{n-1}+\ldots+a_{1} x+a_{0}, a_{n} \neq 0
\end{aligned}
$$

For the interpolation polynomial, its answers can be acquired by substituting the condition of equation (3) for equation (4), and then solving $n+1$ equations of coefficient $a_{i}$. Of several interpolation polynomials, finding the 2nd approximated equation with just the coordinates of three points requires the Lagrange interpolation polynomial. Equation (5) shows the $n$-th Lagrange interpolation polynomial of a point $\left(x_{0}, x_{1}, \cdots, x_{n}\right)[16]$.

$$
l_{i}=\frac{\left(x-x_{0}\right)\left(x-x_{1}\right) \ldots\left(x-x_{i-1}\right)\left(x-x_{i+1}\right) \ldots\left(x-x_{n}\right)}{\left(x_{i}-x_{0}\right)\left(x_{i}-x_{0}\right) \ldots\left(x_{i}-x_{i-1}\right)\left(x_{i}-x_{i+1}\right) \ldots\left(x_{i}-x_{n}\right)}, i=0,1, \ldots, n
$$

When there are coordinate points $\left(x_{0}, y_{0}\right),\left(x_{1}, y_{1}\right) \cdots$ $\left(x_{\mathrm{n}}, y_{\mathrm{n}}\right)$ equation $(6)$ shows their interpolation polynomial.

$$
p(x)=y_{0} l_{0}(x)+y_{1} l_{1}(x)+\cdots+y_{i} l_{i}(x)+\cdots+y_{n} l_{n}(x)
$$

When the interpolation polynomial is acquired directly from those points as in the above expression, the following is the procedure for the simulating algorithm that acquires approximated value in other points.

(1) Input $n, x, x_{i}, y_{i}$

(2) Set $S=0$

(3) Perform the following steps from $K=0$ to $n$. (3.1) Set $T=y_{k}$

(3.2) Perform the following from the $j=0$ of each $\mathrm{k}$ to $\mathrm{m}$.

(3.3) Calculate $S=S+T$

(4) Output $S=p(x)$

\section{EXPERIMENTING BINOCULAR STEREOSCOPIC CAMERA VERGENCE CONTROL}

\section{Analyzing the characteristics of binocular stereo- scopic camera vergence control using acquired dis- parity information}

For an experiment of extracting binocular stereoscopic disparity information, as in Fig. 4, the convergence distance of the stereoscopic camera was fixed at 1,000 $\mathrm{mm}$, and the observed object was moved $100 \mathrm{~mm}$ at a time from $600 \mathrm{~mm}$ to $1,400 \mathrm{~mm}$ to acquire left \& right images, and disparity information was extracted by the method proposed here.

The following results as in table 1 can be acquired by making the distance of the key-object and that of binocular stereoscopic camera different and applying the proposed disparity information-extracting algorithm to the left and right image of a single object inputted from each point. Here, L means the distance between the left-image key-object and the left standard point of full image, $\mathrm{R}$ means the distance between the rightimage key-objectand the left standard point of full image. L-R shows the distance difference between the left standard points of the right and left images of the same key-object, and IL-RI is the absolute value of the acquired distance difference.

As the above Table 1 shows, the difference between the central points of the key-object in its left and right images is gradually decreasing and then increasing. 


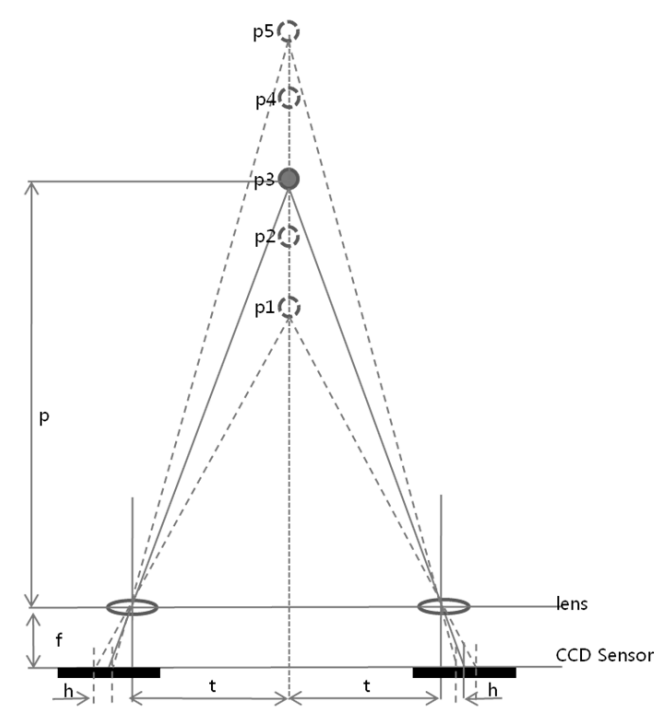

FIG. 4. A design of acquiring left and right image according to distance.

TABLE 1. Disparity information according to the distance of the key-object (convergence distance, $1000 \mathrm{~mm}$ )

\begin{tabular}{c|c|c|c|c}
\hline \hline Distance[mm] & $\mathrm{L}$ & $\mathrm{R}$ & $\mathrm{L}-\mathrm{R}$ & |L-RI \\
\hline 600 & 60 & 73.1 & -13.1 & 13.1 \\
\hline 800 & 72 & 76.4 & -4.4 & 4.4 \\
\hline 1000 & 79.1 & 77.7 & 1.4 & 1.4 \\
\hline 1200 & 84.4 & 79.1 & 5.3 & 5.3 \\
\hline 1400 & 88.1 & 80.6 & 7.5 & 7.5 \\
\hline
\end{tabular}

This means that high quality stereoscopic image can be acquired when the convergence distance of binocular stereoscopic camera is the same as the distance of the key-object that is, the disparity value of the key-object is the minimum. That, in other words, means acquiring the best stereoscopic image from the point of $1,000 \mathrm{~mm}$ where vergence control makes the distance of the key-object and the convergence distance of the camera the same. Therefore, acquiring a stereoscopic image always requires vergence control of the binocular stereoscopic camera according to variance in the distance of the key-object.

\section{Experimenting with vergence control by esti- mating disparity information}

The study acquired a little more complex image through the binocular stereoscopic camera, and extracted disparity information by image processing, and made an experiment on estimation of the disparity value by Lagrange interpolation. As in Fig. 5, disparity information was extracted through the acquired experimental image according to distance, and then the estimated value was analyzed by applying the Lagrange interpolation equation. For the same distance, left image(L) means

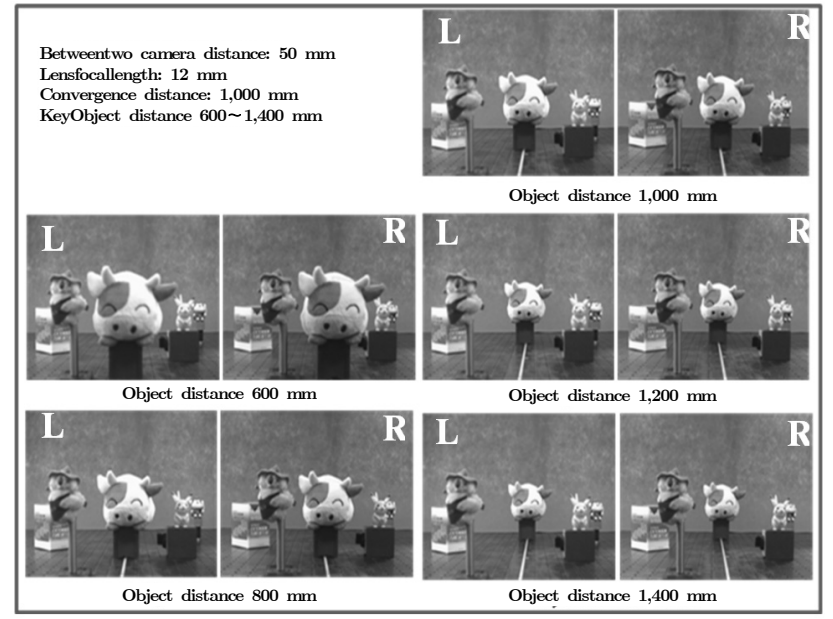

FIG. 5. Acquiring the experimental image of Multi Objects.

left-eye image, and right image $(\mathrm{R})$ means right-eye image. Moving the key-object has caused distance difference between the left image and the right image, which has been compared with the theoretical value by a numerical expression, as in Table 2 .

Table 2 shows the results of extraction and interpolation of disparity information in a complex environment. As for estimation of disparity information, the study assumed that a specific binocular stereoscopic camera already has the disparity information of the key-object on three spots beforehand, and we made an experiment of estimating disparity information by Lagrange interpolation. The study fixed the maximum distance at 1,400 $\mathrm{mm}$, and then calculated the disparity difference of left and right images at a long distance from the key-object and at a short distance and at a middle one, and acquired a Lagrange interpolation equation, based on the former results. As shown in Table 2, experiment Case 1 with 700, 900, and 1,400 $\mathrm{mm}$ as standard distances has proved that vergence control at $1,000 \mathrm{~mm}$ brings the minimum error in real estimated value and theoretical value. And experiment Case 2 with 600,900 , and $1,300 \mathrm{~mm}$ as standard distances also proved that vergence control is fixed at $1,000 \mathrm{~mm}$ corresponded to estimation value. And the error range of theoretical estimated value and real measured value is within about $20 \mathrm{~mm}$ by each distance, which has been found rather accurate. Also, for experiment Case 3(700 $9001,100 \mathrm{~mm})$ establishing all three points as either a long distance or a short distance and experiment Case 4 (1,000 1,200 1,400 mm) establishing all the three points just as a long distance, the theoretical value converges on the minimum distance $(1,000 \mathrm{~mm})$, so the study has proved that any three points can have accurate vergence control. Also, focusing on either a long distance or a short distance can prevent vergence control from going wrong by emphasizing just one of the error range ends. 
TABLE 2. Comparing the estimated value and the real measured value of vergence control according to established three points

\begin{tabular}{c|c|c|c|c|c|c|c|c|c|c|c|c|c|c|c|}
\hline \hline \multirow{2}{*}{ No. } & \multirow{2}{*}{$\begin{array}{c}\text { Object } \\
\text { distance } \\
(\mathrm{mm})\end{array}$} & $\begin{array}{c}\text { Left } \\
\text { image }\end{array}$ & $\begin{array}{c}\text { Right } \\
\text { image }\end{array}$ & L-R & $\begin{array}{c}\text { Absolute } \\
\text { value }\end{array}$ & \multicolumn{2}{|c|}{ Case 1} & \multicolumn{3}{|c|}{ Case 2} & \multicolumn{3}{c}{ Case 3} & \multicolumn{2}{c}{ Case 4} \\
\hline 1 & 600 & 98.3 & 114 & -15.7 & 15.7 & 15.7 & 0.00 & 15.7 & 0.00 & 15.7 & 0.00 & 26.69 & 10.99 \\
\hline 2 & 700 & 104.4 & 114.7 & -10.3 & 10.3 & 11 & 0.70 & 10.91 & 0.61 & 10.96 & 0.66 & 17.16 & 6.86 \\
\hline 3 & 800 & 106.7 & 113.1 & -6.4 & 6.4 & 6.82 & 0.42 & 6.76 & 0.36 & 6.86 & 0.46 & 9.47 & 3.07 \\
\hline 4 & 900 & 110.2 & 113.4 & -3.2 & 3.2 & 3.16 & 0.04 & 3.25 & 0.05 & 3.4 & 0.20 & 3.62 & 0.42 \\
\hline 5 & 1,000 & 112.1 & 112.6 & -0.5 & 0.5 & 0.02 & 0.48 & 0.38 & 0.12 & 0.58 & 0.08 & 0.39 & 0.11 \\
\hline 6 & 1,100 & 114.1 & 112.4 & 1.7 & 1.7 & 2.6 & 0.90 & 1.85 & 0.15 & 1.6 & 0.10 & 2.56 & 0.86 \\
\hline 7 & 1,200 & 114.5 & 111.4 & 3.1 & 3.1 & 4.7 & 1.60 & 3.44 & 0.34 & 3.14 & 0.04 & 2.89 & 0.21 \\
\hline 8 & 1,300 & 116.5 & 111.9 & 4.6 & 4.6 & 6.28 & 1.68 & 4.39 & 0.21 & 4.04 & 0.56 & 1.38 & 3.22 \\
\hline 9 & 1,400 & 118.4 & 112.7 & 5.7 & 5.7 & 7.34 & 1.64 & 4.7 & 1.00 & 4.3 & 1.40 & 1.97 & 3.73 \\
\hline
\end{tabular}

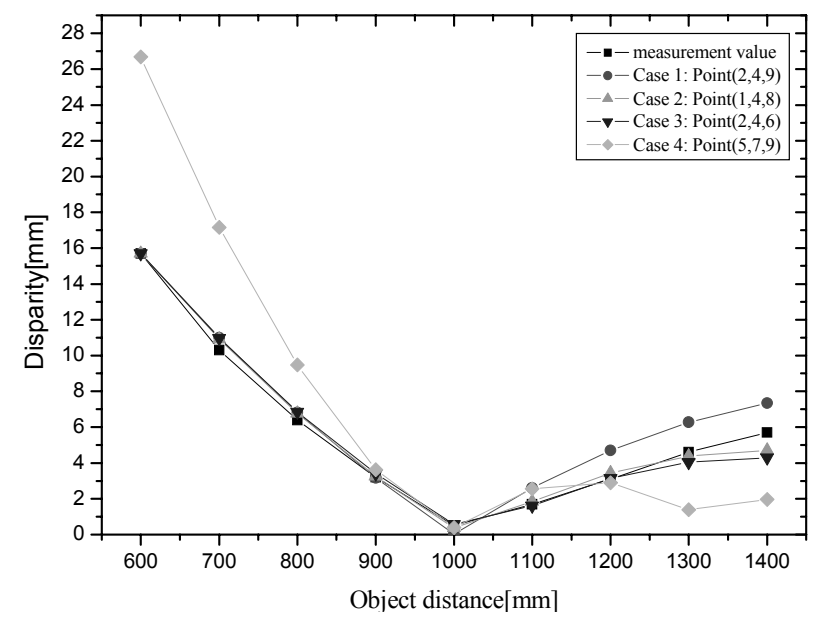

FIG. 6. Variance in the disparity of left and right images according to distance between camera and key-object.

Case 1 : Point 700, 900, 1,100 mm.

Case 2 : Point 600, 900, 1,300 $\mathrm{mm}$.

Case 3 : Point 700, 900, 1,100 mm.

Case 4 : Point 1,000, 1,200, 1,400 mm

The following Fig. 6 shows real measured values based on the data in Table 2 and theoretical values for four cases of interpolation polynomial. It shows that they all converge on $1,000 \mathrm{~mm}$, and that binocular stereoscopic camera vergence control is very similar to the real measured value when the interpolation polynomial is applied to any three long- or short- or middle-distance points.

\section{CONCLUSION}

This study has extracted the disparity information of the key-object for binocular stereoscopic camera vergence control, and then proposed an algorithm calculating the vergence distance of the key-object. The proposed method can be used as algorithm for automatic vergence control of a binocular camera, considered important in optics. The proposed vergence control method extracts the key-object from the acquired stereoscopic data through labeling, and calculates the disparity value of left \& right images. The study took advantage of the disparity information of left and right images obtained by varying distance between camera and the key-object, and could estimate the quantity of vergence control by applying this to the 2nd Lagrange approximated equation. And it could simplify the stereoscopic camera vergence control method by proving that the control points of estimated value and real measured value converge. Therefore, it is expected that the proposed method can be used as automatic algorithm in time of minute adjustment of vergence control.

\section{ACKNOWLEDGMENT}

"This work was supported by the grant of the Korean Ministry of Education, Science and Technology (The Regional Core Research Program/Chungbuk BIT Research-Oriented University Consortium)"

\section{REFERENCES}

1. K. C. Kwon, J. K. Choi, and N. Kim, “Automatic control of horizontal-moving stereoscopic camera by disparity compensation,” J. Opt. Soc. Korea 6, 150-155 (2002).

2. A. A. Dumbreck, E. Abel, and S. P. Murphy, "3-D TV system for remote handling: development and evaluation," Proc. SPIE 1256, 226-236 (1990).

3. S. Y. Park, Y. B. Lee, and S. I. Chien, "Linear relation for vergence control of parallel stereo camera," Electron. Lett. 34, 254-256 (1998). 
4. N. A. Dodgson, "Resampling radially captured images for perspectively correct stereoscopic display," Proc. SPIE 3295, 100-110 (1998).

5. H. Yamanoue, M. Okui, and I. Yuyama, "A study on the relationship between shooting conditions and cardboard effect of stereoscopic images," IEEE Transactions on Circuits and Systems for Video Technology 10, 411-416 (2000).

6. T. C. Wei, D. H. Shin, and B. G. Lee, "Resolutionenhanced reconstruction of 3D object using depth-reversed elemental images for partially occluded object recognition," J. Opt. Soc. Korea 13, 139-145 (2009).

7. M. S. Kim, G. Baasantseren, N. Kim, and J. H. Park, "Hologram generation of 3D objects using multiple orthographic view images,” J. Opt. Soc. Korea 12, 269-274 (2008).

8. A. Wood, T. Docherty, and R. Koch, "Image distortions in stereoscopic video systems," Proc. SPIE 1915, 36-48 (1993).

9. E. Krotkov. K. Henriksen, and R. Kories, "Stereo ranging with verging cameras," IEEE Transactions on Pattern Analysis and Machine Intelligence 12, 1200-1205 (1990).

10. M. Tanaka and N. Maru, "Binocular gaze holding of a moving object with the active stereo vision system," in Proc. IEEE Workshop on Applications. Computer Vision (Sarasota, USA, Dec. 1994), pp. 250-255.

11. H. Hirschmuller, "Improvements in real-time correlation based stereo vision," in Proc. IEEE Workshop on SMBV2001 (Kauai, USA, Dec. 2001), pp. 141-148.

12. D. J. Coombs and C. M. Brown, "Cooperative gaze holding in binocular vision," IEEE Control Systems 11, 24-33 (1991).

13. D. J. Coombs and C. M. Brown, "Real time smooth pursuit tracking for a moving binocular robot," in Proc. Computer Vision and Pattern Recognition (Champaign, USA, Jun. 1992), pp. 23-28.

14. J. R. Taylor, T. Olson, and W. N. Martin, "Accurate vergence control in complex scenes," in Proc. Computer Vision and Pattern Recognition (Seattle, USA, Jun. 1994), pp. 540-545.

15. S. M. Ross, Introduction to Probability and Statistics for Engineers and Scientists (Elsevier Academic Press, Oxford, UK, 2000), Chapter 2.

16. E. Kreysizig, Advanced Engineering Mathematics, 8th ed. (John Wiley \& Sons Inc., New York, USA, 1999), Chapter 17. 